\title{
Prognostic Factors in Operated Cases of Acute Subdural Hematoma
}

\author{
Jaykumar Gunjkar', ${ }^{1}$ SS Vhora², Anindya Gupta ${ }^{3}$ \\ ${ }^{1}$ Assistant Professor, Department of Neurosurgery, BJ Medical College, ${ }^{2}$ Professor, Department of Neurosurgery, BJ Medical \\ College, ${ }^{3}$ Senior Resident, Department of Neurosurgery, BJ Medical College, India
}

Corresponding author: Dr Jaykumar Gunjkar, Richwood Soc, F/501, Spine Road,Chikhli PCNT, Chinchwad, Pune, 411019, Maharashtra, India

DOI: http://dx.doi.org/10.21276/ijcmsr.2019.4.3.12

How to cite this article: Jaykumar Gunjkar, SS Vhora, Anindya Gupta. Prognostic factors in operated cases of acute subdural hematoma. International Journal of Contemporary Medicine Surgery and Radiology. 2019;4(3):C52-C56.

\section{A B S T R A C T}

Introduction: Trauma is a leading cause of mortality in people aged younger than 45 years. Head injury is the number one cause of trauma mortality and it is directly associated with one half of all deaths associated with trauma. Traumatic acute subdural Hematoma is one of the most common traumatic neurosurgical emergencies and often required surgical intervention. This clinical study was done to examine the hospital mortality and analyse the risk factors for mortality in patients treated surgically for isolated traumatic acute subdural Hematoma.

Material and methods: Prospective evaluation of 82 operated cases of ASDH was done. Data was collected from case files and operative records. CT scan variables, clinical variables and operative variables were evaluated.

Results: overall mortality was $30.49 \%$. Male mortality was $31.25 \%$ and female mortality was $27.78 \%$. High mortality with advanced age. No clinically significant difference in mortality due to different mechanism of injury. Other significant mortality indicators were pupillary reaction, midline shift, diffuse edema and hypotension.

Conclusion: The overall mortality of $30.49 \%$ was within the predicted range as per recent literature. Age of the patient, pupillary reaction, Hematoma thickness, midline shift and evidence of diffuse brain swelling during surgery were found to be independent predictors of mortality. This findings may allow for more informed decision making in approaching a particular case.

Keywords: Prognostic Factors, Acute Subdural Hematoma

\section{INTRODUCTION}

Acute subdural Hematoma occurs in 12 to $30 \%$ of patients with severe head injury and reported mortality rates varies from 36 to $79 \%$. In spite of development and improvement in emergency medical service systems and neuro intensive monitoring and treatment, acute subdural Hematoma is still a disorder with very high mortality and extremely poor prognosis among traumatic brain injuries. ${ }^{1-5}$ Therefore identifying reliable prognostic factors for acute subdural Hematoma to improve the surgical result in these patients is important. This clinical study examined the hospital mortality and analyse the risk factors for mortality in patients treated surgically for isolated traumatic acute subdural Hematoma.

\section{MATERIAL AND METHODS}

This was a prospective study done and BJ Medical College and sassoon Hospital between October 2016 to October 2017. 82 patients were included in the study. B J Medical College and sassoon Hospital is a tertiary care center and receive head injury patients from nearby areas. CT scan of the brain was done for all patients of moderate and severe head injury. Brain trauma Foundation guidelines were followed to select patients who will require surgical intervention. Following variables were studied.

Clinical variables: Age, gender, mechanism of injury, preoperative GCS score, preoperative pupillary abnormality, presence of hypotension on admission.

CT scan variables: Maximum thickness of subdural Hematoma, extent of midline shift, presence of associated lesions like contusions and presence of cerebral edema. operative variables: Time elapsed between injury and surgery and evidence of brain swelling during surgery.

The final outcome was calculated as mortality or alive.

\section{RESULTS}

A total of 82 patients were included in the study. Overall mortality was 30.49\%. 64 patients were males and 18 patients were females. Mortality in males was $31.25 \%$ and mortality in females was $27.78 \%$. This difference was not clinically significant. The mortality in less than 40 years age group was $16.66 \%$ that is out of 20 patients 4 patients died. The mortality in 40 to 64 years age group was $22.22 \%$ while the mortality in greater than 65 years age group was $59.09 \%$. This difference in mortality among different age group was found to be clinically significant. Difference in mortality due 


\begin{tabular}{|c|c|c|c|c|c|}
\hline Variable & Total No. of Patients & Alive patients & Dead patient & $\%$ Mortality & P value \\
\hline \multicolumn{6}{|l|}{ Sex } \\
\hline male & 64 & 44 & 20 & $31.25 \%$ & 0.9944 \\
\hline femal & 18 & 13 & 5 & $27.78 \%$ & \\
\hline \multicolumn{6}{|l|}{ AGE } \\
\hline$<40$ & 24 & 20 & 4 & $16.66 \%$ & \\
\hline $40-65$ & 36 & 24 & 8 & $22.22 \%$ & 0.0027 \\
\hline$>65$ & 22 & 9 & 13 & $59.09 \%$ & \\
\hline \multicolumn{6}{|c|}{ Mechanism of injury } \\
\hline MVA & 55 & 38 & 19 & $34.54 \%$ & \\
\hline Fall & 24 & 19 & 5 & $20.83 \%$ & 0.4737 \\
\hline Assault & 3 & 2 & 1 & $33.33 \%$ & \\
\hline \multicolumn{6}{|l|}{ Hypotention } \\
\hline Present & 17 & 9 & 8 & $47.05 \%$ & 0.1703 \\
\hline Not Present & 65 & 48 & 17 & $26.15 \%$ & \\
\hline \multicolumn{6}{|c|}{ Pupilary reaction } \\
\hline Group 1 & 45 & 36 & 9 & $20 \%$ & \\
\hline Group 2 & 24 & 16 & 8 & $33.33 \%$ & 0.0335 \\
\hline Group 3 & 4 & 2 & 2 & $50 \%$ & \\
\hline Group 4 & 9 & 3 & 6 & $66.66 \%$ & \\
\hline \multicolumn{6}{|c|}{ Pre operative GCS } \\
\hline $3-8$ & 36 & 21 & 15 & $41.66 \%$ & \\
\hline $9-11$ & 33 & 25 & 8 & $24.24 \%$ & 0.1269 \\
\hline $13-15$ & 13 & 11 & 2 & $13.38 \%$ & \\
\hline \multicolumn{6}{|c|}{ Hematoma thickness } \\
\hline$<15 \mathrm{~mm}$ & 42 & 33 & 9 & $21.42 \%$ & \\
\hline $15-25 \mathrm{~mm}$ & 31 & 21 & 10 & $32.25 \%$ & 0.0269 \\
\hline$<25 \mathrm{~mm}$ & 9 & 3 & 6 & $66.66 \%$ & \\
\hline \multicolumn{6}{|l|}{ MID line shift } \\
\hline $5-10 \mathrm{~mm}$ & 59 & 47 & 12 & $20.33 \%$ & \\
\hline $11-15 \mathrm{~mm}$ & 18 & 10 & 8 & $44.44 \%$ & \\
\hline$>16 \mathrm{~mm}$ & 5 & 0 & 5 & $100 \%$ & \\
\hline \multicolumn{6}{|c|}{ Associated lesions } \\
\hline Present & 59 & 41 & 18 & $30.50 \%$ & 0.7945 \\
\hline Not Present & 23 & 16 & 7 & $30.40 \%$ & \\
\hline \multicolumn{6}{|l|}{ Time elapsed } \\
\hline$<4 \mathrm{hrs}$ & 36 & 29 & 7 & $19.44 \%$ & \\
\hline 4-8 hrs & 25 & 17 & 8 & $32 \%$ & \\
\hline$>8 \mathrm{hrs}$ & 21 & 11 & 10 & $47.61 \%$ & \\
\hline \multicolumn{6}{|c|}{ Diffuse brain swelling } \\
\hline Present & 23 & 9 & 4 & $60.86 \%$ & \\
\hline Not Present & 59 & 48 & 11 & $35.59 \%$ & \\
\hline
\end{tabular}

to different mechanism of injury that is $34.54 \%$ in patients of MVA, $20.83 \%$ in patients of falls and $33.33 \%$ in patients of assault was not clinically significant. Presence of hypotension was found to be a significant variable in terms of mortality (47\% versus $26 \%$ mortality). Pupillary reaction at the time of admission had a bearing on mortality and it was clinically significant. Similarly lower GCS score on admission was associated with increased mortality.

$41.66 \%$ mortality in patients with GCS score between 3 to 8, 24.24\% mortality in patients with GCS score between 9 to 12 and $13 \%$ mortality in patients with GCS score between 13 to 15 . In the CT scan variables the Hematoma thickness and extent of midline shift was associated with increased mortality. Delay in surgery affected the mortality adversely (19.44\% mortality when delay was less than 4 hours versus $32 \%$ mortality when delay was 4 to 8 hours and $47.67 \%$ mortality when the delay was more than 8 hours). Also the presence of diffuse cerebral edema which was evaluated intra operatively in the form of presence of herniation of the brain parenchyma craniectomy site was found to be significantly associated with mortality (6.86 percent mortality versus $35.59 \%$ mortality).

\section{DISCUSSION}

India has rather unique distinction of having the highest number of head injuries in the world. In India more than 
1 lakh lives are lost every year with over 1 million suffering from serious head injury. ${ }^{1}$ ASDH is one of the most common surgical emergencies and often required surgical intervention. In this study we attempted to study the parameters affecting the outcome and mortality in patients of ASDH. 82 patients of traumatic acute subdural hematomas were included in the study who were admitted and operated under department of neurosurgery of BJ medical college between the period of October 2016 to October 2017. We observed a hospital mortality rate of $30.49 \%$ in 82 patients with isolated traumatic acute subdural hematomas. These figures are less than the mortality of $36 \%$ to $79 \%$ which is reported in the literature..$^{2-9}$ This may be due to improvements in transfer service facilities by the EMRI services sponsored by government, emergency management at the site of accident, widespread availability of modern imaging modalities in most secondary and tertiary centers, availability of neurosurgeons 24 hours in hospital, acceptance of the philosophy of improvements in results due to early surgery, improved anaesthesia management and improved post operative Intensive Care Management. Out of 82 patients included in the study 64(78\%) were males and $18(22 \%)$ were females. Mortality among males was $31.25 \%$ and a mortality among females was $27 \%$. This difference was not statistically significant. All over the world males outnumber females in relation to incidence of acute subdural hematomas. ${ }^{9-18}$ The age distribution of traumatic brain injury across the various series revealed that highest occurrence is in the age group of 20 to 29 years followed by 30 to 39 years. ${ }^{19-27}$ In our study those patients younger than 40 years had mortality rate of $16.66 \%$. Where as patients in the age group 40 to 64 years had a mortality rate of $22.22 \%$. The increased mortality in the elderly patients may be partly explained by the intrinsic properties of the aging brain, pre-existing comorbidities and complications. ${ }^{28-32}$ Further experimental studies have shown that the ageing brain is more susceptible to deleterious effects of ischemia, which is a common pathophysological mechanism underlying severe form of traumatic brain injury. ${ }^{33-37,5}$ Furthermore the adverse effects of general anaesthesia and surgery may affect the respiratory and circulatory functions of the elderly increasing the severity of brain injury. The influence of trauma etiology and mortality has been reported in earlier studies. Wilburg et $\mathrm{al}^{29}$ found no significant difference in mortality and functional recovery in trauma due to various causes. Steaning et $\mathrm{al}^{37}$ demonstrated that road traffic victims had a higher mortality rate when compared with other accidents. In our study there was no statistically significant difference in mortality due to various causes. With regards to factors influencing outcome, the impact of GCS score has been studied most frequently. The most detailed analysis of the effect of GCS score on outcome after severe traumatic brain injury was done in the IMPACT (International mission for prognosis and analysis of clinical trials in TBI) ${ }^{10}$ study. It was shown that the GCS score on Hospital admission was strongly related to the GOS score at 6 months after trauma. In our study high mortality of $41.66 \%$ was seen in group of patients with GCS score of 3to8. In GCS score 9 to 12 the mortality was $24.24 \%$ which further decreased to $15.38 \%$ in patients with GCS score of 13 to 15 . This association between GCS score and mortality was not found to be statistically significant in our study, one of the reasons for this might be the less sample size in a particular group as per the GCS score and mortality. This Results are confirmed by the study of Koc et al. ${ }^{25}$ Gennarelli et $\mathrm{al}^{26}$ published mortality rate of $74 \%$ for Patients with acute SDH and GCS score of 3-5, and 36\% for those with GCS score between 6-10. In our study the mortality was found to vary according to the reactivity of the pupils as follows, normal symmetrical pupillary reaction the mortality was $25 \%$, asymmetrical pupils one reacting the mortality was $33.33 \%$, asymmetrical pupils both pupils not reacting the mortality was $50 \%$, bilateral fixed dilated pupils the mortality was $66.66 \%$. Pupillary reaction and Pupil size correlates with injury to the brain stem. Many authors reported that patients with bilateral fixed pupils at surgery had favorable outcome in only $0-13 \%$ only and mortality rate $64-93 \% .11,23,27,32$ In our study patients with systolic blood pressure less than $80 \mathrm{~mm}$ of Mercury on admission were categorized as hypotension present and the remaining as hypotension absent.

Presence of hypotension leads to secondary brain injury and increased mortality. In our study there was $47.05 \%$ mortality in patients with hypotension versus $26.15 \%$ mortality in patient without hypotension. Johannes leitgeb, walter mauritz ${ }^{28}$ in their recent study on prognostic factors in ASDH found statistically significant association between mortality and prehospital hypotension (70\% versus 44\% mortality). Actually there are few other studies which have emphasized on this factor. In our study there was a steep increase in mortality farm $21.42 \%$ in group of patients with Hematoma thickness more than 15 millimetres to $66.66 \%$ for patients with Hematoma thickness more than $25 \mathrm{~mm}$. Yanka et $\mathrm{al}^{29}$ showed that the mean Hematoma volume was $31 \mathrm{cc}$ for those patients with a favourable recovery. Similarly it was $104 \mathrm{cc}$ for those cases with a poor outcome. Stone et $\mathrm{al}^{30}$ demonstrated that patients with the Hematoma volume of less than hundred cc had a favourable recovery in $32 \%$ and mortality rate of $51 \%$ while those cases harbouring Hematoma with volume over hundred cc had only $8 \%$ favourable outcome and mortality rate of $79 \%$.

Larger the midline shift worse is the outcome. The degree of midline shift is determined by the volume of the acute subdural Hematoma + the swelling of the underlying hemisphere. Marshall et $\mathrm{al}^{32}$ reported that greater midline shift was associated with a worse recovery. Ross et $\mathrm{al}^{33}$ demonstrated a trend towards high mortality if the midline shift is more

than $5 \mathrm{~mm}$. Becker et $\mathrm{al}^{34}$ concluded that the mortality in patients with $10 \mathrm{~mm}$ or greater midline shift was $53 \%$ when compared with $25 \%$ for those with midline shift of 5 to 10 $\mathrm{mm}$. The reported incidence of brain contusions associated with an acute subdural Hematoma ranges from $7 \%{ }^{11}$ to $82 \% .{ }^{4}$ This wide variability probably reflects difference in both causes of trauma and time from injury to CT scan. The presence of associated brain contusions is a powerful predictor of bad outcome. Infact the rate of a favourable outcome ranges from 58 to $32 \%$ for patients with an isolated acute subdural Hematoma and from 19 to $12 \%$ for those with associated multiple brain contusions. ${ }^{4,31}$ All tough much has been learnt about brain swelling associated with acute 
subdural Hematoma in the past decade the fundamental reasons that why it occurs after traumatic brain injury and the factors that are responsible have not been identified. The pathophysiology of traumatic acute subdural Hematoma is based not only on the volume of the Hematoma but also the severity and the distribution of the primary traumatic insert and the presence of secondary insult. acute subdural Hematoma with underlying brain swelling implies that the hemisphere beneath the lesion may have suffered more focal parenchymal damage compared to the contralateral side. In our study the outcome of acute subdural Hematoma patients with brain swelling was poor even when treated with early surgical evacuation. it is possible that poor outcome depends not only on the Hematoma itself but also on the presence of cerebral parenchyma injury and secondary insults. It must be emphasized that the development of neuroprotective drugs therapy to reduce brain oedema formation is essential for improving the outcome in acute subdural Hematoma patients with brain swelling. Out of the many variables that have been found to significantly correlated either positively or negatively with morbidity and mortality from acute subdural hematomas only two can potentially be affected by neuro surgical intervention they are the time from injury to operative intervention for evacuation of Hematoma and the control of ICP. Prompt surgical intervention for evacuation of traumatic intracranial Hematoma have been emphasize in the literature but little hard evidence is available to supports its efficacy. Most of the emphasis has been on prompt evacuation of epidural Hematoma. In our study there was the exponential increase in mortality from $19 \%$ to $47 \%$ in patients operated within less than four hours to patients operated more than 8 hours after.

\section{CONCLUSIONS}

Time elapsed between trauma and definative management in the form of surgical evacuation of Hematoma is not only important predictor of outcome but it is also one of the factors which is modifiable. Timeliness depends upon a wellintegrated prehospital system with trained paramedics and established region wise protocols as well as rapid access to definitely imaging and surgical interventions. Through this paper we would like to stress that the establishment of a comprehensive trauma system at the state regional and country level is an independent determinant of outcome in trauma and a laudable Public Health goal.

\section{REFERENCES}

1. Epidemiology of traumatic brain injuries. Indian Scenario. Gururaj G. Neurol Res. 2002

2. Bullock R, Sakas D, Patterson J, et al. Early posttraumatic cerebral blood flow mappin: Correlating with structural damage after focal injury. Acta Neurochir (Wien). 1992;55(1):14-7.

3. Deb S, Bhaumik S, Pal H. Isodense acute subdural hematoma in anemic patients. Neural India. 2000;48:298-9.

4. Faiz Ahmad U, Mahapatra AK. Intraoperative development of posterior fossa extradural hematoma during evacuation of frontal contusion. Neurol India. 2006;54 (3): 219-20.
5. Prognosis of ASDH in the elderly: A systematic review. Evans LR. J neurotrauma 2019

6. Lamieson KG, Yelland ID. Surgically treated traumatic sub-dura! hematomas. J Neurosurg. 1972;37 (5):137-49.

7. Jones N, Bullock R, Reilly P. The role of surgery for intracranial mass lesions after head injury. In: Reilly $\mathrm{P}$, Bullock R (Eds). Head Injury. London: Chapman and Hall Medical; 1997. pp. 419-21.

8. Kotwica Z, Brzezinski J. Acute subdural hematoma in adults: an analysis of outcome in comatose patients. Acta Neurochirur (Wien). 1993;121 (6):95-9.

9. Kristiarisen K, Tandon PN. Diagnosis and treatment of severe craniocerebral injuries. J Oslo City Hosp (Suppl). 1960;10 (3);107- 213.

10. Impact Study. Roozenbeek B. crit care Med. 2012.

11. Matfiew P, Oluoch-Olunya DL, Condon BR, et al. Acute subdural haematoma in conscious patients: outcome with initial non- operative management. Acta Neurochirur (Wien). 1993;121 (2):100-8.

12. Maxciner H, Wolff M. Pure subdural hematomas: a postmorterm analysis of their form and bleeding points. Neurosurgery. 2002;50:503-9.

13. Mendelow AD, Teasdale CM, Russell T, et al. Effect of mannitol on cerebral blood flow and cerebral perfusion pressure in human head injury. I Neurosung. 1985;63 (1):43-8.

14. Miller JD, Bullock R, Graham DI, et al. Ischemic brain damage in a model of acute subdural hermatoma. Neurosurgery. 1990;27 (5):433- 9.

15. Ramamurthi B. Acute subdural haematoma. In: Vinken PI, Bruyn and Skull. Amsterdam: North Holland Publishing Company; 1976.

16. Servadei F. Prognostic factors in severly head injured adults with ASDH. Acta Neurochir (Wien). 1997;139(4):279-85.

17. Servadei F, Nasi MT, Giuliani G, Cremonini AM, Cenni P, Zappi D, Taylor GS. CT prognostic factors in acute subdural haematomas: the value of the 'worst' CT scan. Br J Neurosurg. 2000;14(2):110-6.

18. Stone J et al. Subdural hematomas. I. Acute subdural hematoma: progress in definition, clinical pathology, and therapy. Surg Neurol. 1983;19(3):216-31.

19. Tandon PN. ASDH -A Reappraisal. Neurology India 2001;49 (6):3-10

20. Teasdale E. Head injury Pathophysiology and management of severe closed injury. J Neurol Neurosurg Psychiatry. 2006; 77(5): 710.

21. Valadka AB, Robertson CS. Surgery of cerebral trauma and associated critical care. Neurosurgery. 2007;61(1 Suppl):203-20.

22. Van Den Brink. The Prognostic Importance of the volume of $\mathrm{EDH}$ and SDH. Acta neurochirur. 1995;23 (1):23-29.

23. Wong CW. Criteria for conservative treatment of ASDH. Acta Neurochir (Wien). 1995;135(1-2):38-43.

24. Zumkellerm. CT Criteria for survival rate for patients with ASDH. Neurosurgery. 1996;23 (6):34-39.

25. Koch N. Out come And out come Predictions in ASDH. Surg neurol. 1993

26. Gennareli TA. Influence of the type of intra cranial lesion on out come of severe head injury. J Neurosurg 1982. 
27. Stening WA. Experience in ASDH in new south wales. Aust Nz J Surg. 1986;12 (2):23-29.

28. Out come after severe head injury due to ASDH. Johnannes Leitjeb. J Neurosurg 2012;21 (3):34-39

29. Yanaka K.ASDH - Prediction of out come with a linear discriminant function. Neural med chir Tokyo. 1993;3 (1):34-39.

30. Stone JL. ASDH: Direct Admission To a trauma center yields better results. J Trauma 1986;10 (6):23-29.

31. Epidemiology of traumatic head injury. Indian scenario. Indian J of Epidemiology and Statistics 2006.

32. Marshall lf. The national traumatic coma data bank, Part 2 J neurosurg. 1983

33. Ross AI. Prediction of out come in brain injury with CT characteristics. Neurosurgery 2005;23 (5):23-29.

34. Becker DP. Out come from severe head injury with early diagnosis and intensive management. J neurosurg. 1977;23 (1):34-39.

35. Road traffic deaths, injuries and disabilities in India: current scenario, Gururaj G. Department of Epidemiology, WHO collaborating Centre for Injury Prevention and Safety Promotion, national Institute of Mental Health and Neuro Sciences, Bangalore560029, Karnataka, India. Natl Med J India. 2008;21(1):14-20.

Source of Support: Nil; Conflict of Interest: None

Submitted: 05-06-2019; Accepted: 18-07-2019; Published online: 25-07-2019 